\title{
PROGRAMAS DE ORDENADOR BASADOS EN MINDFULNESS. UNA REVISIÓN DE LA LITERATURA MÉDICA
}

\author{
Alba López-Montoyo ${ }^{\mathrm{a}}$ \\ Av. Compromiso de Caspe $931^{\circ} \mathrm{C} 50002$, Zaragoza \\ allomo91@gmail.com \\ ${ }^{a}$ Universidad Jaume I, Castellón, España \\ Marta Modrego-Alarcón ${ }^{b}$ \\ martamodal@gmail.com \\ ${ }^{b}$ Universidad de Zaragoza, Zaragoza, España \\ Héctor Morillo ${ }^{\mathrm{b}}$ \\ hmorillosarto@gmail.com \\ ${ }^{b}$ Universidad de Zaragoza, Zaragoza, España \\ Javier García-Campayo ${ }^{\mathrm{b}}$ \\ jgarcamp@gmail.com \\ ${ }^{b}$ Universidad de Zaragoza, Zaragoza, España
}

Soledad Quero,

squero@psb.uji.es

${ }^{a}$ Universidad Jaume I, Castellón, España
${ }^{\mathrm{c} C I B E R}$ de Fisiopatología de la Obesidad y Nutrición (CIBEROBN)

\section{RESUMEN}

Introducción: Numerosos estudios han evaluado los efectos de la meditación obteniendo efectos beneficiosos para la salud (Chen, Berger, Manheimer, Forde, Magidson, Dachman y Lejuez, 2012). Internet está suponiendo un nuevo modo de ofrecer programas de tratamiento (Cuijpers, Driessen, Hollon, van Oppen, Barth y Andersson, 2012). El objetivo fue llevar a cabo una revisión de las características y la efectividad de las psicoterapias online basadas en mindfulness. 
Método: Esta revisión se realizó en las bases de datos, excluyéndose los artículos de opinión y los que no tenían una evaluación pre y post tratamiento.

Resultados: Se encontraron un total de 12 artículos. La mayoría de los estudios mostraron una reducción significativa en los síntomas clínicos.

Conclusiones: La aplicación de las terapias basadas en mindfulness a través de Internet puede ayudar a la reducción de la sintomatología clínica y a la mejora del bienestar. Aun así, se hace necesaria más investigación al respecto.

Palabras clave: mindfulness, Internet, síntomas clínicos, online y psicoterapia.

JEL: I10, I19

\begin{abstract}
Background: Many studies have evaluated the effects of meditation and have found positive health benefits (Chen, Berger, Manheimer, Forde, Magidson, Dachman y Lejuez, 2012). Internet means a new way to provide treatment programs (Cuijpers, Driessen, Hollon, van Oppen, Barth y Andersson, 2012). The main aim of the present study is to conduct a review of the characteristics and the effectiveness of the computerassisted psychoterapies mindfulness based.
\end{abstract}

Method: The searches were made in the data bases, excluding opinion articles and articles where a pre-post treatment evaluation was not included.

Results: A total of 12 articles were found. Most of these studies showed a significant reduction of clinical symptoms.

Conclusions: The application of mindfulness based therapies over the Internet can help to reduce clinical symptoms and improve the wellness. However, more research in this regard is needed.

Key words: mindfulness, Internet, clinical symptoms, online and psychotherapy.

JEL: I10, I19

\title{
1. INTRODUCCIÓN
}


Según Jon Kabat-Zinn (1979), Mindfulness o atención plena es "simplemente parar y estar presente". Se caracteriza por dos componentes. El primero de ellos es la autorregulación de la atención que es mantenida sobre la experiencia inmediata, permitiendo así el reconocimiento de los eventos mentales en el momento presente. El segundo componente es la orientación particular hacia la propia experiencia, que se caracteriza por la curiosidad, la apertura y la aceptación (Bishop, Lau, Shapiro, Carlson, Anderson, Cardomy, Segal, Abbey, Speca, Velting y Devins, 2004).

Mindfulness trata de que la persona sea capaz de concentrarse en el presente, es por ello que su eficacia tanto en el tratamiento de la depresión (en la que las personas constantemente están preocupadas acerca de su pasado) y de la ansiedad (que se caracteriza por una preocupación excesiva en el futuro) es alta (Toneatto y Nguyen, 2007; Yeoungsuk y Lindquist, 2015). En el caso concreto del trastorno depresivo, existe amplia evidencia, en forma de meta-análisis, que avala la eficacia de este tipo de intervención (Hoffman, Sawyer, Witt y Oh, 2010). De hecho, la Mindfulness-Based Cognitive Therapy (MBCT) está recomendada por la NICE británica como tratamiento de elección en la depresión recurrente (NICE, 2009).

Se ha demostrado que la psicoterapia de mindfulness mejora la salud mental y física, reduce el afecto negativo y mejora la adaptación a situaciones adversas o estresantes (Chen et al., 2012; Grosman, Niemann, Schmidt, y Walach, 2004; Keng, Smoski, y Robins, 2011). De hecho, en la literatura actual existen diversas revisiones sobre los resultados clínicos de las intervenciones que utilizan el entrenamiento de la atención plena y concluyen que las intervenciones basadas en Mindfulness conducen a efectos beneficiosos en una gran variedad de condiciones médicas y psicológicas, a pesar de que se requieren más investigaciones que confirmen y amplíen los hallazgos obtenidos hasta el momento. Las patologías más estudiadas han sido la ansiedad, la depresión, el dolor crónico, la fibromialgia y el síndrome de la fatiga crónica (Allen et al, 2006; Baer, 2003; Bishop, 2002; Dimidjiany Linehan, 2003; Grossman et al, 2004; Hayes, Luoma, Bond, Masuda, y Lillis, 2006; Lazar, 2005; Salmon, Sephton, Weissbecker, Hoover, Ulmer y Studts 2004; Toneatto y Nguyen, 2007).

Por otro lado, Internet ejerce una gran influencia en la sociedad, habiéndose incrementado y ampliado su uso en la actualidad. En el ámbito de la terapia, supone un 
nuevo modo de dispensar programas de tratamiento (Andersson, 2009) a través de lecciones, módulos y tareas que los pacientes deben completar regularmente.

Las revisiones sistemáticas que se han realizado muestran que los tratamientos a través de Internet y los tratamientos computarizados son intervenciones eficaces en distintos trastornos (Cuijpers, Van Straten, Van Oppen y Andersson, 2008). Aunque se han desarrollado distintas psicoterapias a través de Internet (p.ej., psicodinámicas e interpersonales), la mayor investigación se centra en la terapia cognitivo-conductual. Estudios que comparan su eficacia, en comparación con la terapia cognitivo-conductual cara a cara, muestran que ambas son igualmente eficaces en diferentes problemas como el trastorno de ansiedad social, el trastorno de pánico, los síntomas depresivos, la insatisfacción corporal, los zumbidos en los oídos, la disfunción sexual masculina y la fobia a las arañas. No obstante, se necesitan más estudios que ayuden a confirmar estos resultados (Andersson, Cuijpers, Carlbring, Riper y Hedman, 2014).

El uso de Internet tiene una serie de ventajas que hacen que esta tecnología pueda resultar atractiva. Algunas de estas ventajas son la posibilidad de incrementar la frecuencia de contacto con los usuarios, de diseñar programas más personalizados e interactivos o de mantener y potenciar la motivación y la adherencia a las prescripciones terapéuticas a largo plazo (Botella, Baños, García-Palacios y Quero, 2011). También requiere una menor cantidad de recursos clínicos y puede llegar a pacientes que no recibirían tratamiento de otro modo, bien sea, por ejemplo, debido al estigma social asociado a recibir atención por un problema de salud mental, hora y lugar de accesibilidad, coste económico del tratamiento o la escasez de profesionales de salud mental debidamente capacitados para administrar el tratamiento basado en la evidencia (Frewen, Rogers, Flodrowski y Lanius, 2015). Se trata así de una alternativa prometedora para diseminar los tratamientos psicológicos basados en la evidencia (Johansson y Andersson, 2012). A pesar de ello, actualmente, en el proceso de difusión de las intervenciones basadas en Internet en el que nos encontramos, quedan varios desafíos y retos por resolver. Por ejemplo, diversos estudios han manifestado una actitud negativa por parte de pacientes y clínicos hacia la utilización de estos programas de tratamiento (véase para más información en Andersson y Titov, 2014).

En definitiva, la utilización de programas de tratamiento basados en Internet se plantea como una estrategia que puede ser de gran ayuda. No obstante, a pesar de que el número 
de estudios está creciendo, sigue siendo necesaria una mayor investigación, en especial en lo que se refiere a programas a través de Internet que estén basados en mindfulness. El objetivo principal del presente estudio es llevar a cabo una revisión de las características y la efectividad de las psicoterapias por ordenador que contienen mindfulness como componente principal.

\section{MÉTODO}

2.1.Criterios de selección: Esta revisión se basa principalmente en artículos y trabajos originales que han sido publicados y que incluyen específicamente el componente de mindfulness aplicado a través del ordenador, ya sea por medio de una página web propia, o vía e-mail. Nuestro objetivo fue incluir todos los tipos de participantes en el estudio, tanto a población general como a población clínica. Se incluyó cualquier programa de cualquier parte del mundo, lo que significa que aceptamos cualquier idioma (español, inglés, chino...), aunque principalmente los programas encontrados estaban en inglés. Además, los estudios seleccionados tenían que tener una evaluación de la sintomatología previa al entrenamiento y una evaluación posterior al mismo, para observar diferencias pre-post tratamiento, independientemente de si los resultados habían sido una mejora en los síntomas o una ausencia de la misma.

2.2.Estrategia de búsqueda: Se realizaron búsquedas a través de las bases de datos, principalmente Pubmed, Medlinde, Psycarticles y Scholar.google. Se utilizaron las palabras clave y los siguientes operadores:"OR" ("mindfulness" "mindful" "mindfulness" "Mind-body") “AND” (“OR" "ICTs" “TICs" "Internet" "Internet-based" "Computer" "Online" "Web"). Estas bases de datos proporcionan información sobre la investigación de carácter multidisciplinar que involucra a los campos de las ciencias médicas, psicológicas, y de las nuevas tecnologías. En la búsqueda no se estableció ningún criterio anual porque queríamos encontrar lo que había en la literatura en su totalidad, debido a que la introducción de la práctica de mindfulness a través de las nuevas tecnologías es muy reciente. 
2.3.Procedimiento: En primer lugar, se revisaron y seleccionaron los artículos encontrados a través del título y abstract, seleccionando un total de 17 artículos. En segundo lugar, estos 17 artículos fueron revisados y leídos en su totalidad, seleccionando un total de 12 artículos. Se descartaron 5 por no cumplir criterios de inclusión (uno por falta de una evaluación previa y posterior a la aplicación del programa, dos de ellos estaban reclutando pacientes en el momento actual y, por lo tanto, eran estudios activos, y otros dos eran artículos de opinión). Finalmente, de estos 12 artículos se revisó la bibliografía por si podíamos detectar algún otro estudio. Solo uno fue añadido por título y abstract pero, tras su lectura completa, fue descartado debido a que el programa no se aplicaba a través del ordenador sino de forma tradicional.

\section{RESULTADOS}

Se encontraron 12 artículos que cumplían los criterios establecidos y que incluían datos de eficacia de los programas de mindfulness a través de Internet. De estos doce, 10 tenían una página web propia, creada especialmente para uso psicoterapéutico, en la que se incluían herramientas concretas para la práctica de la atención plena y psicoeducación que introducía el trastorno que se trabajaba. Estos programas estaban diseñados para tratar una amplia gama de trastornos, concretamente los artículos seleccionados se dirigían a problemas tales como el trastorno bipolar, fumadores, síntomas depresivos, ansiedad, dolor crónico y síndrome del intestino irritable.

También se encontró 1 curso online con el mismo objetivo, en el que se utilizaban diferentes recursos tecnológicos. Este curso también tenía una página web concreta, donde se ofrecían clases en video (cómo se podría ofrecer en un curso cara a cara) que los participantes debían ir viendo de manera semanal. Además, los ejercicios estaban dirigidos a iniciar a los usuarios en la práctica de mindfulness, ofreciendo esta técnica como herramienta para aumentar el bienestar, o disminuir posibles momentos de ansiedad y no tanto como un tratamiento breve y psicoterapéutico.

Finalmente, en un segundo curso encontrado, la información relevante se enviaba exclusivamente por e-mail, no utilizaban una página web concreta, sino que todos los materiales se envían a través del correo electrónico. 
Por otra parte, todos los programas revisados presentaban elementos comunes en sus métodos, la mayoría utilizaba elementos interactivos como son los vídeos, flashes animados, textos, ejercicios a realizar, archivos de audio, imágenes, manuales de aclaración e información y ejemplos explicativos. Las sesiones se realizaban semanalmente. La duración de los programas variaba, pero se encontraba en torno a las 4-9 sesiones, dependiendo del programa. Los ejercicios comunes que se realizaba eran: el Body Scan, Mindfulness en la respiración, práctica de los tres minutos, una comida Mindfulness, Mindfulness caminando, se trabajan las prácticas informales y, en algunos artículos, se realizaban prácticas adaptadas al trastorno al que iba dirigido el programa.

Por lo que se refiere a los resultados de eficacia informados en estos estudios, éstos fueron diferentes. Así, algunos mostraban una disminución significativa de los síntomas clínicos, en otros estudios no había significación, otros solo vieron reducidas únicamente algunas variables medidas, mientras que otras se mantuvieron estables. A pesar de ello, se observa que en la mayoría de los estudios la sintomatología tiende a reducirse, sobre todo en lo que se refiere a síntomas ansiosos, depresivos y al aumento del bienestar, la atención y la conciencia. Toda la información referente a los estudios y programas existentes la podemos encontrar más detallada en la Tabla 1 .

\section{DISCUSIÓN}

Algunas terapias utilizan distintos programas online para aplicar sus técnicas, obteniendo importantes ventajas y quedando demostrada su eficacia en diferentes trastornos. En lo que se refiere a mindfulness, este formato de aplicación comienza a cobrar importancia.

El objetivo del presente estudio fue llevar a cabo una revisión de las características y la efectividad de las psicoterapias por ordenador que contienen mindfulness como componente principal. La mayoría de los estudios encontrado se dirigían a la sintomatología de diversos trastornos, como la ansiedad y la depresión, lo que no es de extrañar, dado que existen muchos estudios que apoyan la eficacia de las técnicas de mindfulness sobre este perfil sintomático. En cuanto a la efectividad de los programas, es importante subrayar que los estudios encontrados presentaban limitaciones metodológicas, entre las que destacan, por un lado, la falta de grupos controles que permitan realizar comparaciones entre grupos, la utilización de cuestionarios no validados y el empleo de muestras muy reducidas o centradas únicamente en un perfil 
de la población. Todo ello dificulta extrapolar los resultados a la población a la que se dirija el programa en cuestión. Por otro lado, el tratamiento farmacológico que recibían algunos participantes no fue tomado en consideración en el análisis de los resultados. Por último, en este tipo de estudios existe un gran porcentaje de abandonos durante el proceso de seguimiento, e incluso en algunas de las investigaciones dichos seguimientos ni siquiera se llevaron a cabo, lo que impide saber cuál es el efecto de estos programas a largo plazo.

Las intervenciones breves que utilizan específicamente mindfulness a través de las nuevas tecnologías pueden ser útiles para facilitar el acercamiento a la práctica, la adherencia al entrenamiento, e incluso para reducir determinados síntomas clínicos. Los resultados encontrados en esta revisión van en la línea de lo esperado, y aunque muestran resultados prometedores, todavía existen pocos estudios y muchas limitaciones, sobre todo en lo que se refiere a la prevención de los síntomas más que al tratamiento de los mismos. La gran mayoría de los estudios revisados están orientados a reducir la sintomatología clínica, excepto dos de ellos que están dirigidos a aumentar el bienestar y, por lo tanto, a prevenir posibles síntomas futuros. Es necesario realizar más estudios controlados que apoyen la eficacia de intervenciones de mindfulness a través de las nuevas tecnologías, puesto que no se han desarrollado apenas investigaciones, hasta la fecha, con rigor metodológico.

La revisión bibliográfica de este artículo se ha realizado a partir de una búsqueda narrativa y no sistemática, por lo que algunos programas de ordenador que estén fundamentados en mindfulness, pueden no haberse incluido. Asimismo, el incremento del número de recursos bibliográficos podría haber llevado a un mayor número de hallazgos de programas basados en Mindfulness.

Las Tecnologías de la Información y la Comunicación están evolucionando rápidamente, lo que ha favorecido un mayor acercamiento de los tratamientos a la población, además de un mayor interés en el estudio científico de este tipo de intervenciones. Como líneas futuras de investigación en este campo, se están elaborando programas que utilizan los teléfonos móviles y la realidad virtual (RV) como soporte para la aplicación de técnicas de intervención, beneficiándose así de las ventajas que ofrecen, como son la inmediatez y comodidad en el caso de los smarthphones y la inmersión en el caso de la RV. 
Por lo que se refiere a las aplicaciones móviles, sus características permiten el acceso a la información y datos en cualquier lugar y momentoy, además, pueden ser transportados fácilmente gracias a su pequeño tamaño, a esto se le añade la gran capacidad de procesamiento, conexión de red y amplia memoria (Plaza, Demarzo, Herrera-Mercadal, y García-Campayo, 2013), facilitando su adaptación e inclusión en las actividades del día a día. A este respecto, destaca la revisión realizada por, Plaza, Piva, Herrera-Mercadal y García-Campayo (2013) donde se pusieron de manifiesto las aplicaciones móviles existentes hasta la fecha que estaban basadas en mindfulness y técnicas meditativas. Aunque se encontró una amplia cantidad de las mismas a disposición de las personas interesadas, no existía evidencia empírica que apoyara su utilidad. Resultados similares fueron encontrados más recientemente por Mani, Kavanagh, Hides y Stoyanov (2015). Por tanto, es necesario realizar más investigaciones controladas al respecto con el fin de encontrar una posible evidencia científica que respalde el uso de estas aplicaciones y su efectividad.

Por otro lado, por lo que se refiere a RV, en la literatura revisada se contemplan algunos estudios en los que se han creado entornos virtuales basados en mindfulness, aunque el número de ellos es muy escaso. Un ejemplo de estos entornos virtuales es el "Virtual Meditative Walk", un sistema construido mediante RV, tecnología de biofeedback y la técnica Mindfulness-Based Stress Reduction (MBSR) que resulta eficaz en la reducción de dolor percibido, en pacientes con dolor crónico (Gromala, Tong, Choo, Karamnejad, y Shaw, 2015). Kosunen et al. (2016) también presentaron el sistema "Relaworld", un sistema de RV en meditación que combina la RV con neurofeedback. Los resultados de este estudio, realizado en estudiantes universitarios, indicaron que el sistema permite una relajación más profunda, una mayor sensación de presencia y un nivel más profundo de meditación.

Se puede decir que la RV está demostrando ser una herramienta valiosa, como procedimiento de tratamiento en sí mismo, y para aumentar la utilidad clínica de técnicas de intervención cognitivo-comportamentales (Botella, García-Palacios, Baños y Quero, 2007). Sin embargo, como se manifiesta en la literatura, existen muy pocos estudios que relacionen RV y mindfulness $\mathrm{y}$, al igual que ocurre con las aplicaciones móviles, es necesario realizar más investigaciones controladas que respalden su uso y efectividad. 
Hoy en día la psicoterapia ha de adaptarse a los nuevos tiempos para poder llegar de una forma óptima al máximo número de personas que se puedan ver beneficiadas de ella. Entre estos avances, las nuevas tecnologías y su integración en la vida cotidiana favorecen que determinadas herramientas como Mindfulness puedan ser desarrolladas a través de un software informático.

A medida que se avanza en la investigación sobre mindfulness, es lógico que se vayan explorando todas las plataformas posibles para su expansión. El tiempo y la investigación seguirá arrojando luz sobre la eficacia de los programas online de mindfulness como ya se ha demostrado esta eficacia en programas online enfocados en la terapia cognitivo conductual en diversas psicopatologías.

El hecho de que las personas continuamente convivan con las nuevas tecnologías hace fácil su adherencia y su acceso a un bajo coste, trayendo consigo tanto rigurosidad en los contenidos, como disponibilidad a la hora de acceder a los tratamientos.

Bien es cierto que aunque las nuevas tecnologías nos brindan estas ventajas respecto a un modelo de tratamiento presencial, la investigación sobre la eficacia de estos programas aún está por determinar, siendo necesarios más estudios al respecto. No obstante es evidente que las líneas futuras de investigación y tratamiento en psicología deberían ir ligadas al uso de las nuevas tecnologías. 


\section{REFERENCIAS}

Academic Mindfulness Interest Group, M., \& Academic Mindfulness Interest Group, M. (2006). Mindfulness-based psychotherapies: a review of conceptual foundations, empirical evidence and practical considerations. Australian and New Zealand Journal of Psychiatry, 40(4), 285-294.

Andersson, G. \& Titov, N. (2014). Advantages and limitation of Internet-based interventions for common mental disorders. World Psychiatry, 13, 4-11.

Andersson, G. (2009). Using the Internet to provide cognitive behaviour therapy. Behaviour Research and Therapy, 47,175-80.

Andersson, G., Cuijpers, P., Carlbring, P., Riper, H., \& Hedman, E. (2014). Guided Internet-based vs. face-to-face cognitive behavior therapy for psychiatric and somatic disorders: a systematic review and meta-analysis. World Psychiatry, 13(3), 288-295.

Baer, R. A. (2003). Mindfulness training as a clinical intervention: A conceptual and empirical review. Clinical psychology: Science and practice, 10(2), 125-143.

Berman, R. L. H., Iris, M. A., Bode, R., \& Drengenberg, C. (2009). The Effectiveness of an Online Mind-Body Intervention for Older Adults With Chronic Pain. The Journal of Pain, 10(1), 68-79.

Bishop, S. R. (2002). What do we really know about mindfulness-based stress reduction?. Psychosomatic medicine, 64(1), 71-83.

Bishop, S. R., Lau, M., Shapiro, S., Carlson, L., Anderson, N., Cardomy, J., Segal, Z., Abbey, S., Speca, M., Velting, D. \& Devins, G. (2004). Mindfulness: A proposed operational definition. Clinical Psychology: Science and Practice, 10, 230- 241

Boettcher, J., Åström, V., Påhlsson, D., Schenström, O., Andersson, G., \& Carlbring, P. (2014). Internet-Based Mindfulness Treatment for Anxiety Disorders: A Randomized Controlled Trial. Behavior Therapy, 45(2), 241-253. 
Boggs, J. M., Beck, A., Felder, J. N., Dimidjian, S., Metcalf, C. A., \& Segal, Z. V. (2014). Web-Based Intervention in Mindfulness Meditation for Reducing Residual Depressive Symptoms and Relapse Prophylaxis: A Qualitative Study. Journal of Medical Internet Research, 16(3), e87.

Botella, C., Baños, R, García-Palacios, A., y Quero, S. (2011). Nuevas tecnologías al servicio de la intervención. En Técnicas de Modificación de Conducta (pp.659-671). Madrid: Ediciones Pirámide.

Botella, C., García-Palacios, A., \& Baños, R. M. y Quero, S. (2007). Realidad virtual y tratamientos psicológicos. Cuadernos de medicina psicosomática y psiquiatría de enlace, (82), 35-46.

Burns, M. N., Begale, M., Duffecy, J., Gergle, D., Karr, C. J., Giangrande, E., \& Mohr, D. C. (2011). Harnessing context sensing to develop a mobile intervention for depression. Journal of medical Internet research, 13(3), e55.

Chen, K. W., Berger, C. C., Manheimer, E., Forde, D., Magidson, J., Dachman, L., \& Lejuez, C. W. (2012). Meditative therapies for reducing anxiety: a systematic review and meta-analysis of randomized controlled trials. Depression and anxiety, 29(7), 545562.

Chittaro, L., \& Vianello, A. (2014). Computer-supported mindfulness: evaluation of a mobile thought distancing application on naive meditators. International Journal of Human-Computer Studies, 72(3), 337-348.

Choi, I., Zou, J., Titov, N. et al. (2012). Culturally attuned Internet treatment for depression amongst Chinese Australians: a randomised controlled trial. Journal of Affective Disorders, 136, 459-68.

Cuijpers, P., Driessen, E., Hollon, S. D., van Oppen, P., Barth, J., \& Andersson, G. (2012). The efficacy of non-directive supportive therapy for adult depression: A metaanalysis. Clinical Psychology Review, 32(4), 280-291. 
Cuijpers, P., van Straten, A., Andersson, G., \& van Oppen, P. (2008). Psychotherapy for depression in adults: a meta-analysis of comparative outcome studies. Journal of consulting and clinical psychology, 76(6), 909.

Davis, J. M., Manley, A. R., Goldberg, S. B., Stankevitz, K. A., \& Smith, S. S. (2015). Mindfulness training for smokers via web-based video instruction with phone support: a prospective observational study. BMC Complementary and Alternative Medicine, 15(1), 95.

Dilts, R., \& Hallbom T, S. S. (1990). Beliefs: Pathways to Health \&amp; Well Being.

Frewen, P., Rogers, N., Flodrowski, L., \& Lanius, R. (2015). Mindfulness and Mettabased Trauma Therapy (MMTT): Initial Development and Proof-of-Concept of an Internet Resource. Mindfulness, 6(6), 1322-1334.

Garrison, K. A., Pal, P., Rojiani, R., Dallery, J., O’Malley, S. S., \& Brewer, J. A. (2015). A randomized controlled trial of smartphone-based mindfulness training for smoking cessation: a study protocol. BMC Psychiatry, 15, 83.

Glück, T. M., \& Maercker, A. (2011). A randomized controlled pilot study of a brief web-based mindfulness training. BMC Psychiatry, 11(1), 175.

Gromala, D., Tong, X., Choo, A., Karamnejad, M., \& Shaw, C. D. (2015, April). The virtual meditative walk: Virtual reality therapy for chronic pain management. In Proceedings of the 33rd Annual ACM Conference on Human Factors in Computing Systems (pp. 521-524).

Grossman, P., Niemann, L., Schmidt, S., \& Walach, H. (2004). Mindfulness-based stress reduction and health benefits: A meta-analysis. Journal of psychosomatic research, 57(1), 35-43.

Hayes, S. C., Luoma, J. B., Bond, F. W., Masuda, A., \& Lillis, J. (2006). Acceptance and commitment therapy: Model, processes and outcomes. Behaviour research and therapy, 44(1), 1-25. 
Hofmann, S. G., Sawyer, A. T., Witt, A. A., \& Oh, D. (2010). The effect of mindfulness-based therapy on anxiety and depression: A meta-analytic review. Journal of consulting and clinical psychology, 78(2), 169.

Johansson, R., \& Andersson, G. (2014). Internet-based psychological treatments for depression. Expert review of neurotherapeutics.

Kabat-Zinn, J. (2003). Vivir con plenitud las crisis. Cómo utilizar la sabiduría del cuerpo y la mente para afrontar el estrés, el dolor y la enfermedad. Barcelona: Kairós.

Kemper, K. J., \& Yun, J. (2015). Group online mindfulness training: proof of concept. Journal of Evidence-Based Complementary \& Alternative Medicine, 20(1), 73-5.

Keng, S. L., Smoski, M. J. y Robins, C. J. (2011). Effects of mindfulness on psychological health: a review of empirical studies. Clinical Psychology Review, 31(6).

Kosunen, I., Salminen, M., Järvelä, S., Ruonala, A., Ravaja, N., \& Jacucci, G. (2016, March). RelaWorld: Neuroadaptive and Immersive Virtual Reality Meditation System. In Proceedings of the 21st International Conference on Intelligent User Interfaces (pp. 208-217). ACM.

Krusche, A., Cyhlarova, E., \& Williams, J. M. G. (2013). Mindfulness online: an evaluation of the feasibility of a web-based mindfulness course for stress, anxiety and depression. BMJ Open, 3(11).

Krusche, A., Cyhlarova, E., King, S., \& Williams, J. M. G. (2012). Mindfulness online: a preliminary evaluation of the feasibility of a web-based mindfulness course and the impact on stress. BMJ Open, 2(3).

Krusche, A., Cylharova, E. y Williams, J. M. G. (2013). Mindfulness online: an evaluation of the feasibility of a web based mindfulness course for stress, anxiety and depression. British Medical Journal Open, 3(11), 1-10 
Lazar, S. W., Kerr, C. E., Wasserman, R. H., Gray, J. R., Greve, D. N., Treadway, M. T., ... \& Rauch, S. L. (2005). Meditation experience is associated with increased cortical thickness. Neuroreport, 16(17), 1893.

Levis, D. (1997). ¿Qué es la realidad virtual. Recuperado de http://www. diegolevis. com. ar/secciones/Articulos/Que_es_RV.Pdf

Ljótsson, B., Falk, L., Vesterlund, A. W., Hedman, E., Lindfors, P., Rück, C., ... Andersson, G. (2010). Internet-delivered exposure and mindfulness based therapy for irritable bowel syndrome - A randomized controlled trial. Behaviour Research and Therapy, 48(6), 531-539.

Mak, W. W., Chan, A. T., Cheung, E. Y., Lin, C. L., \& Ngai, K. C. (2015). Enhancing Web-Based Mindfulness Training for Mental Health Promotion With the Health Action Process Approach: Randomized Controlled Trial. Journal of Medical Internet Research, $17(1)$

Mani, M., Kavanagh, D. J., Hides, L., \& Stoyanov, S. R. (2015). Review and Evaluation of Mindfulness-Based iPhone Apps. JMIR mHealth and uHealth, 3(3), e82.

Meyer, B., Berger, T., Caspar, F., Beevers, C. G., Andersson, G., \& Weiss, M. (2009). Effectiveness of a Novel Integrative Online Treatment for Depression (Deprexis): Randomized Controlled Trial. Journal of Medical Internet Research, 11(2), e15.

Miller G (2012). The Smartphone Psychology Manifesto. Perspectives on Psychological Science, 7(3):221-237.

Mira Pastor, A., Botella Arbona, C., Riera López del Amo, A., Moragrega Vergara, I., \& Soler Rovira, C. (2015). Un programa de Tratamiento de corte CognitivoComportamental basado en las TIC para la prevención y el tratamiento de la depresión: Descripción del programa y datos preliminares. Àgora de Salut, (1), 283-297.

Monshat, K., Vella-Brodrick, D., Burns, J., \& Herrman, H. (2012). Mental health promotion in the Internet age: a consultation with Australian young people to inform the 
design of an online mindfulness training programme. Health Promotion International, $27(2), 177-186$.

Morledge, T. J., Allexandre, D., Fox, E., Fu, A. Z., Higashi, M. K., Kruzikas, D. T., ... Reese, P. R. (2013). Feasibility of an Online Mindfulness Program for Stress Management-A Randomized, Controlled Trial. Annals of Behavioral Medicine, 46(2), $137-148$.

Murray, G., Leitan, N. D., Berk, M., Thomas, N., Michalak, E., Berk, L., ... Kyrios, M. (2015). Online mindfulness-based intervention for late-stage bipolar disorder: pilot evidence for feasibility and effectiveness. Journal of Affective Disorders, 178, 46-51.

Pérez, M. A., \& Botella, L. (2007). Conciencia plena (mindfulness) y psicoterapia: concepto, evaluación y aplicaciones clínicas. Revista de psicoterapia, 17(66-67), 77120.

Plaza, I., Piva, M., Herrera-Mercadal, P. y García-Campayo, J. (2013). MindfulnessBased Mobile Applications: Literature Review and Analysis of Current Features. JMIR mhealth and whealth, 1(2).

Salmon, P., Sephton, S., Weissbecker, I., Hoover, K., Ulmer, C., \& Studts, J. L. (2004). Mindfulness meditation in clinical practice. Cognitive and behavioral practice, 11(4), 434-446.

Simón, V. (2007). Mindfulness y neurobiología [Mindfulness and neurobiology]. Revista de Psicoterapia, 66/67, 5-30.

Song, Y., \& Lindquist, R. (2015). Effects of mindfulness-based stress reduction on depression, anxiety, stress and mindfulness in Korean nursing students. Nurse education today, 35(1), 86-90.

The National Institute for Health and Clinical Excellence (NICE). (2009). Depression in Adults. NICE clinical guidelines. 
Toneatto, T., \& Nguyen, L. (2007). Does mindfulness meditation improve anxiety and mood symptoms? A review of the controlled research. Canadian Journal of Psychiatry, 52(4), 260.

Veehof, M. M., Oskam, M. J., Schreurs, K. M. y Bohlmeijer, E. T. (2011). Acceptancebased interventions for the treatment of chronic pain: a systematic review and metaanalysis. Journal of Pain, 152(3), 533-542.

Wagner, B., Schulz, W. y Knaevelsrud, C. (2012). Efficacy of an Internet- based intervention for posttraumatic stress disorder in Iraq: a pilot study. Psychiatry Research, $195,85-8$.

Younge, J. O., Wery, M. F., Gotink, R. A., Utens, E. M. W. J., Michels, M., Rizopoulos, D., ... Roos-Hesselink, J. W. (2015). Web-Based Mindfulness Intervention in Heart Disease: A Randomized Controlled Trial. PLOS ONE, 10(12), e0143843.

Zernicke, K. A., Campbell, T. S., Speca, M., McCabe-Ruff, K., Flowers, S., Dirkse, D. A., \& Carlson, L. E. (2013). The eCALM Trial-eTherapy for cancer appLying mindfulness: online mindfulness-based cancer recovery program for underserved individuals living with cancer in Alberta: protocol development for a randomized waitlist controlled clinical trial. BMC Complementary and Alternative Medicine, 13(1), 34. 
Tabla 1. Programas de Ordenador con Mindfulness encontrados en la literatura.

\begin{tabular}{|c|c|c|c|c|c|c|c|c|}
\hline & Programa & Año & Descripción & Eficacia/resultados & Programa & Año & Descripción & Eficacia/Resultados \\
\hline \multirow{5}{*}{ 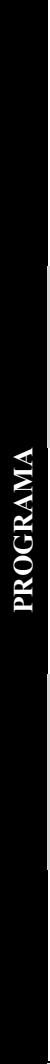 } & ORBIT & 2015 & $\begin{array}{l}\text { Programa breve y de baja intensidad } \\
\text { basado en mindfulness para } \\
\text { Trastorno Bipolar }\end{array}$ & $\begin{array}{c}\text { Mejora de calidad de vida } \\
\text { subjetiva }\end{array}$ & $\begin{array}{c}\text { Internet-delivered } \\
\text { exposure and } \\
\text { mindfulness based } \\
\text { therapy for irritable } \\
\text { bowel Syndrome. A } \\
\text { randomized } \\
\text { controlled trial }\end{array}$ & 2011 & $\begin{array}{l}\text { Programa dirigido a pacientes } \\
\text { de Síndrome del Intestino } \\
\text { Irritable, basado en la } \\
\text { exposición y mindfulness } \\
\text { mensajes en línea, textos } \\
\text { escritos y páginas web. Acceso } \\
\text { a un foro de discusión en línea. }\end{array}$ & $\begin{array}{c}\text { La exposición y la atención } \\
\text { plena a través de Internet } \\
\text { puede ser eficaz en el } \\
\text { tratamiento de pacientes } \\
\text { con SII, aliviando la carga } \\
\text { total de los síntomas y el } \\
\text { aumento de la calidad de } \\
\text { vida. }\end{array}$ \\
\hline & $\begin{array}{l}\text { Web-Based } \\
\text { Mindfulness Training } \\
\text { for Mental Health } \\
\text { Promotion }\end{array}$ & 2015 & $\begin{array}{c}\text { Se evalúa la eficacia de dos } \\
\text { programas uno de mindfulness básico } \\
\text { y otro de mindfulness HAPA. Basado } \\
\text { en las técnicas del MBSR. Para } \\
\text { población General. }\end{array}$ & $\begin{array}{l}\text { Los participantes de ambos } \\
\text { programas mostraron una } \\
\text { mejora del bienestar mental, } \\
\text { atención y en la satisfacción } \\
\text { con la vida. }\end{array}$ & $\begin{array}{l}\text { Online Mindfulness } \\
\text { Program for Stress } \\
\text { Management (ISM) }\end{array}$ & 2013 & $\begin{array}{l}\text { Programa autodirigido, } \\
\text { accesible a través de la Web o } \\
\text { un hipervínculo, para personas } \\
\text { con Ansiedad. }\end{array}$ & $\begin{array}{l}\text { Mejora en la percepción } \\
\text { del estrés, la tensión } \\
\text { arterial, la atención, y la } \\
\text { autoaceptación. }\end{array}$ \\
\hline & $\begin{array}{c}\text { Web-Based } \\
\text { Mindfulness } \\
\text { Intervention in Heart } \\
\text { Disease }\end{array}$ & 2015 & $\begin{array}{l}\text { Se enseñan diferentes ejercicios de } \\
\text { mindfulness, autoreflexión y yoga a } \\
\text { pacientes diagnosticados de } \\
\text { Enfermedades Cardíacas. }\end{array}$ & $\begin{array}{c}\text { Reducción de la tasa cardíaca y } \\
\text { de los síntomas de depresión y } \\
\text { ansiedad. No diferencias } \\
\text { significativas en bienestar } \\
\text { psicológico. }\end{array}$ & $\begin{array}{l}\text { Effectiveness of } \\
\text { online mind-body } \\
\text { intervention for older } \\
\text { adults with chronic } \\
\text { pain }\end{array}$ & 2009 & $\begin{array}{l}\text { Programa orientado al auto } \\
\text { cuidado para personas con } \\
\text { Dolor Crónico. }\end{array}$ & $\begin{array}{l}\text { Reducción de la conciencia } \\
\text { de dolor, intensidad del } \\
\text { dolor y la interferencia del } \\
\text { mismo. Aumento de la } \\
\text { confianza en el uso de } \\
\text { técnicas de auto-cuidado. }\end{array}$ \\
\hline & $\begin{array}{l}\text { Mindful Mood } \\
\text { Balance (MMB) }\end{array}$ & 2014 & $\begin{array}{l}\text { Programa online con apoyo por vía } \\
\text { telefónica o vía e mail. Para personas } \\
\text { con Síntomas Depresivos } \\
\text { Residuales. }\end{array}$ & $\begin{array}{l}\text { Mayor conciencia de las } \\
\text { emociones, reducción del } \\
\text { estrés, síntomas depresivos y su } \\
\text { impacto en el comportamiento. }\end{array}$ & DEPREXIS & 2009 & $\begin{array}{l}\text { Programa online basado en la } \\
\text { activación comportamental, } \\
\text { restructuración cognitiva, } \\
\text { ejercicios de mindfulness y } \\
\text { entrenamiento en habilidades } \\
\text { sociales. Para Depresión }\end{array}$ & $\begin{array}{c}\text { Reducción de la } \\
\text { sintomatología depresiva y } \\
\text { mejora en habilidades } \\
\text { sociales. Las mejoras se } \\
\text { mantuvieron a los meses. }\end{array}$ \\
\hline & $\begin{array}{c}\text { Tratamiento basado } \\
\text { en mindfulness para } \\
\text { los trastornos de } \\
\text { ansiedad. }\end{array}$ & 2014 & $\begin{array}{l}\text { Mindfulness para Ansiedad. } \\
\text { Se les daba acceso a la web y al } \\
\text { finalizar se les enviaba un e-mail. }\end{array}$ & $\begin{array}{c}\text { Reducción de síntomas } \\
\text { ansiosos, incluyendo síntomas } \\
\text { depresivos y de insomnio }\end{array}$ & $\begin{array}{l}\text { Mindfulness Training } \\
\text { for Smokers Online } \\
\text { (MTSO) }\end{array}$ & 2015 & $\begin{array}{l}\text { Tiene una página web, se les } \\
\text { ofrece un usuario y contraseña. } \\
\text { Clases en vídeo, apoyo } \\
\text { telefónico semanal y se les } \\
\text { proporciona un manual. } \\
\text { Para Fumadores }\end{array}$ & $\begin{array}{c}\text { Los participantes } \\
\text { mostraron una reducción } \\
\text { significativa de la ansiedad } \\
\text { y un aumento de } \\
\text { mindfulness. }\end{array}$ \\
\hline$\frac{8}{8}$ & $\begin{array}{l}\text { Web-based } \\
\text { mindfulness course } \\
\text { for stress, anxiety } \\
\text { and depression }\end{array}$ & 2013 & $\begin{array}{l}\text { Meditación guiada, vídeos, trabajos y } \\
\text { e-mails con elementos del MBSR y } \\
\text { del MBCT. Consiste en } 10 \text { sesiones } \\
\text { interactivas dirigidas a Población en } \\
\text { General }\end{array}$ & $\begin{array}{c}\text { Reducción en el estrés } \\
\text { percibido y en los posibles } \\
\text { síntomas ansiosos y depresivos } \\
\text { de forma significativa. }\end{array}$ & & & & \\
\hline$\sum_{\substack{1 \\
\mid=1}}^{E}$ & $\begin{array}{c}\text { A randomized } \\
\text { controlled pilot } \\
\text { study of a briefweb- } \\
\text { based mindfulness } \\
\text { training (2011). }\end{array}$ & 2011 & $\begin{array}{c}\text { Entrenamiento basado en mindfulness } \\
\text { vía e-mail para adultos con diferentes } \\
\text { niveles de Ansiedad. Dos módulos en } \\
13 \text { días. Se enviaban archivos de } \\
\text { audio, flashes animados, ejercicios y } \\
\text { textos escritos. }\end{array}$ & $\begin{array}{l}\text { Efectos en el estrés percibido, } \\
\text { en el afecto negativo a largo } \\
\text { plazo y en el afecto positivo a } \\
\text { medio plazo. Efectos similares } \\
\text { en la reducción de estrés con } \\
\text { cara a cara. }\end{array}$ & & & & \\
\hline
\end{tabular}


